\title{
Electroluminescent Polymer Materials and Their Applications
}

\author{
Xinjie Tao ${ }^{1,2}$, Yongli $\mathrm{Li}^{1,2}$, Yuechan $\mathrm{Li}^{1,2}$, Dongya Sun ${ }^{1,2^{*}}$, An $\mathrm{Xie}^{1,2}$ \\ ${ }^{1}$ Key Laboratory of Functional Materials and Applications of Fujian Province, Xiamen University of Technology, Xiamen \\ 361024, China \\ ${ }^{2}$ School of Materials Science and Engineering, Xiamen University of Technology, Xiamen 361024, China
}

Corresponding Author Email: 2013123205@xmut.edu.cn

https://doi.org/10.18280/acsm.450306

Received: 12 January 2021

Accepted: 26 April 2021

\section{Keywords:}

electroluminescence, high polymer materials, panel display

\begin{abstract}
This paper mainly introduces the light-emitting mechanism, preparation technology, and application status of electroluminescent polymer materials. As an important category of conductive polymer, electroluminescent polymer materials are also known as organic electroluminescent (EL) diode materials or organic light-emitting diode (OLED) materials. Compared with traditional display materials and devices, OLED materials boast a high light-emitting efficiency, an easily adjustable wavelength, a long service life, and an excellent machining performance. In the light of these advantages, this paper summarizes the progresses and future trends of OLED materials and devices in green lighting and panel display.
\end{abstract}

\section{INTRODUCTION}

Organic polymer photoelectric materials have received extensive attention from researchers, thanks to its broad application prospects [1-10]. For many years, scientists have studied the synthesis, structure, conductive mechanism, performance, and application of conductive polymer. The recent progress in these areas has elevated conductive polymer to an independent discipline.

In some fields, the dominance of liquid crystal displays (LCDs) in the panel display market is being challenged by organic light-emitting diode (OLED) display. Compared with LCD panel display, OLED panel display is an efficient and energy-saving lighting device. There are many advantages with OLED panel display: active light emission, thinness and lightweight, brilliant colors, full-angle view, and low energy consumption. As a result, many research institutes, leading electronics manufacturers, and world-famous chemical companies have invested lots of manpower and money to OLED research [11-15]. Despite the enormous success in the research, there is still room for further improvement in terms of overall light-emitting efficiency, wavelength adjustment, stability, and service life.

This paper reviews the recent progress of OLED materials and devices in terms of preparation process and quality, as well as the main problems with these materials and devices.

\section{DEVICES AND PRINCIPLE OF OLED}

Electroluminescence refers to the light emission directly excited by electric energy. Figure 1 shows the principle of electroluminescence: the holes and electrons injected by electrodes migrate in the target material, and combine into excitons (excited molecules); then, the electrons jump from the conduction band of excitons to the holes in the valence band; the excess energy is released in the form of light, hence the light-emission phenomenon.
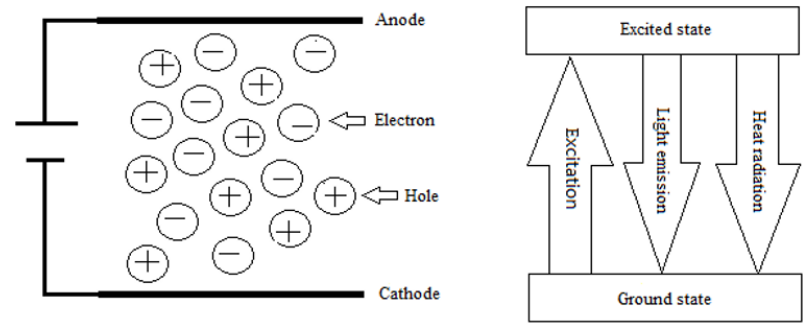

Figure 1. Principle of electroluminescence

Currently, there are three basic structures for electroluminescent polymer devices (Figure 2).

(a)
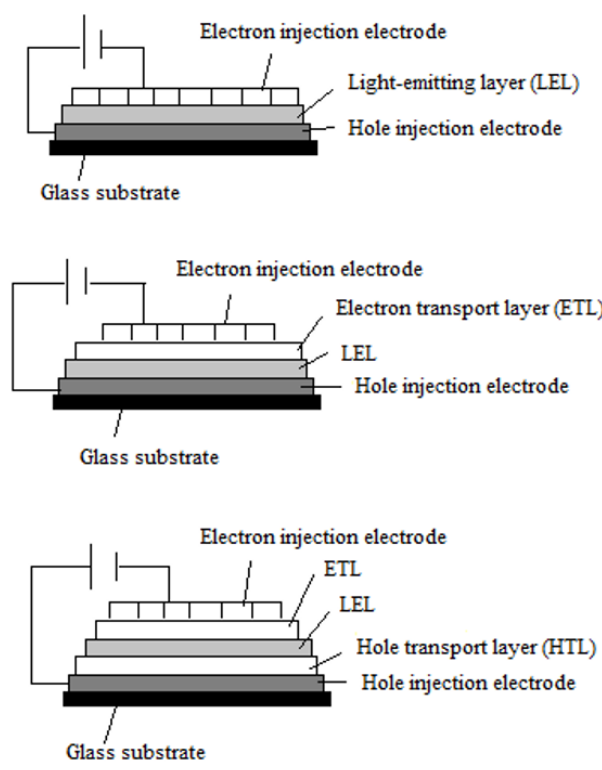

Figure 2. Structures of electroluminescent polymer devices 
Figure $2 \mathrm{a}$ is a simple sandwich structure: an LEL is sandwiched between an electron injection electrode and a hole injection electrode. This structure can be prepared in the following steps: spin coating, dip coating or vacuum steaming light-emitting material (LEL) on the conductive glass substrate; plating with cathode material; connecting the power source.

To improve the stability and efficiency of the OLED device, the injection of electrons should be balanced with the injection of holes. This means the power function of the electrode material should match the energy level of the electroluminescent material. The general practice is to add an ETL or an HTL to the system (Figure 2(b)) or add both ETL and HTL to the system (Figure 2(c)).

The charge transport layers aim to balance electron transport with hole transport, such that the two carriers could combine precisely in the LEL into light-emitting excitons. Each charge transport layer facilitates the transport of one kind of charges, and hinders that of the other. In general, the ETL blocks the transport of holes, while the HTL blocks the transport of electrons.

To facilitate the emission of visible light, one of electron injection electrode and hole injection electrode must be transparent. The multilayer structure helps to balance electrode injection with hole injection, and improve the performance of OLED devices. Experiments have shown that OLED devices with multilayer structure manage to achieve a low driving voltage, and a balance between electron injection and hole injection. Both contribute to the composite probability of excitons and quantum efficiency of electroluminescence.

\section{TYPES OF OLED MATERIALS}

\subsection{Charge injection materials}

\subsubsection{Electron injection material}

The main function of electron injection material is to inject negative charges into the electroluminescent material. The desired features of electron injection material include good conductivity, suitable work function, and desirable physicalchemical stability. The material should transmit the driving voltage evenly to the organic material interface, and overcome the interface potential to inject electrons effectively to the organic layer. The commonly used electron injection materials include pure metal, alloy, and metal composite. Alkaline earths and aluminum alloys are the most popular electron injection materials. The oxides or fluorides of these metals can effectively improve electron injection efficiency, namely, $\mathrm{Li}_{2} \mathrm{O}, \mathrm{Al}_{2} \mathrm{O}_{3}, \mathrm{CsCO}_{3}$, and $\mathrm{CsF}$ [16-22].

\subsubsection{Hole injection material}

Apart from hole injection, the anode material must ensure the transparency of the electrode, such that the generated visible light can pass through the electrode. The most widely used anode material is indium-tin oxide (ITO) glass electrode, which has an excellent hole injection performance for most organic matters. The ITO is an ideal hole injection material, with a high transparency and a high work function (4.9). The hole injection performance of the ITO can be significantly enhanced by altering its surface morphology and physicalchemical properties. The alteration is generally realized through proper treatment and chemical modification, using plasma, acid, self-assembled film, or additional buffer layer.
For example, acid treatment can increase the work function by protonating the surface. The most effective acid is phosphoric acid, which can increase the work function by $0.7 \mathrm{eV}[23,24]$.

Besides the ITO, many other metal oxides with high work function, transmittance, and conductivity can serve as hole injection material, namely, fluorine-doped tin oxide (FTO), aluminum-doped zinc oxide (AZO), gallium-doped indium oxide (GIO), gallium and indium-doped tin oxide (GITO), zinc-doped tin oxide (ZIO), and zinc and indium-doped tin oxide (ZITO) [25]. Conjugated polymers can also be prepared into hole injection anode. For instance, replacing the ITO with polyaniline could greatly improve the performance of OLED devices: the operating voltage could drop by $30 \%$, and the quantum efficiency could increase by $10-30 \%$ [26]

\subsection{Charge transmission materials}

The transport materials of carriers can be divided into hole transport material and electron transport material. Generally, hole transporting material is an electron-rich compound, while electron transport material is an electron-deficient compound. Polymer hole transport material is introduced in details below, owing to its extensive application.

\subsubsection{Electron transfer material}

The popular organic electron transport materials include metal complexes and n-type organic semiconductor materials. The favorite complexes among electroluminescence researchers are tris(8-hydroxyquinoline) aluminum ( $\left.\mathrm{Alq}_{3}\right)$ complex and its derivatives. Apart from the good electron transport ability of $\mathrm{Alq}_{3}$, there are many other reasons for the popularity of the complex and its derivatives: $\mathrm{Alq}_{3}$ is easy to synthesize and passivate; the complex enjoys excellent thermal and morphological stability; it can be turned into a film easily through evaporation; the molecular structure prevents the formation of exciplex. However, $\mathrm{Alq}_{3}$ is not ideal in terms of electron mobility, quantum efficiency, and energy gap. Similar complexes have also been frequently adopted for electron transport, including tris(5-hydroxymethyl-8quinolinolato) aluminum (AlOq) [27], bis(5,7-dichloro-8quinolinolato)-(8-quinolinolato)aluminum [Alq(CLq) 2 ] [28], and salicylidene-o-aminophenolato) (8-quinolinoato) aluminum [Al(Saph-q)] [29].

\subsubsection{Hole transport material}

To facilitate hole transport and injection, hole transporting material should have a good nucleophilic property, and a conduction band energy level that matches the anode. Adachi et al. prepared a two-layer LED: ITO / HTL/ emission layer (EML) / Mg: Ag, where the HTL was made of 14 small aromatic amine molecules, and the EML was made of a material with a low ionization potential $(508 \mathrm{eV})$. The materials significantly enhance the stability of the device. Adachi et al. held that the device is more stable with the decline of the energy barrier between the HTL and the anode. In fact, the energy barrier has a great impact on the performance of OLED devices. It was reported that the service life of continuously running devices depends on the energy barrier, i.e., the difference between the ionization potential of the hole transport material and the work function of the ITO. Meanwhile, the thermal stability of OLED devices is closely associated with the glass transition temperature of hole transport material. Therefore, hole transport material must carry the following features: good film-forming property, 
good hole transport ability, and high glass transition temperature.

Polyethylene carbazole (PVK), a typical optical conductor, has been widely adopted as a hole transport material. On the structure of the material, the $\mathrm{N}$ electrophilic atoms absorb the electrons on the double bond through the inductive effect. Due to $\mathrm{p} \pi$ conjugation, the lone pairs of the $\mathrm{N}$ atoms are supplied to the double bond, making it rich in electrons. The conjugation effect is greater than the inducive effect. As a result, PVK has a strong ability of hole transport. In organic electroluminescent (EL) devices, PVK is often used in the HTL. On the one hand, PVK reduces the crystallization of small EL molecules, extending the service life of the device; on the other hand, PVK increases the probability of electronhole composite, and improves the light-emitting efficiency of the device.

Polysilane has attracted much attention for its high charge mobility. This material carries many properties of $\pi$ conjugated polymers, due to the $\sigma$-conjugation in the silicon skeleton. The photoconductivity of polymers is determined by light-emitting property and charge mobility. To enhance the sensitivity within the visible range, substituent groups should be connected to the side link. Polymethylsilane (PMPS) is a favorable hole transport material. At room temperature, its hole mobility is $10^{-3} \mathrm{~cm} / \mathrm{Vs}$. It is easy to obtain and process pure PMPS samples, which has no absorption within visible light. The material can be wet sprayed onto LEDs.

\subsection{Light-emitting materials}

\subsubsection{Organic small molecule light-emitting materials}

To achieve organic electroluminescence, organic small molecule light-emitting materials need to satisfy the following requirements: (1) strong fluorescence and no obvious concentration quenching in solid state; (2) strong transport ability of carriers; (3) good thermal and chemical stability; (4) supporting vacuum evaporation.

As shown in Figure 3, $\mathrm{Alq}_{3}$ is the most widely used organic small molecule light-emitting material. It has the advantages of good film-forming property, high carrier mobility, and desired stability. $\mathrm{Alq}_{3}$ is both an electroluminescent material, and an electron transport material. Hence, the material can serve as the ETL in LEDs. Hamada et al. synthesized multiple complexes from three ligands (8-hydroxyquinoline and its two derivatives) and four complex ions $\left(\mathrm{Al}^{3+}, \mathrm{Mg}^{2+}, \mathrm{Zn}^{2+}\right.$ and $\left.\mathrm{Be}^{2+}\right)$ Under the bias voltage of $20 \mathrm{~V}, 8$-hydroxyquinoline $\left(\mathrm{Znq}_{2}\right)$ achieved an illuminance of up to $16,200 \mathrm{~cd} / \mathrm{m}^{2} . \mathrm{Znq}_{2}$ and other two-coordinate metal complexes are expected to become new organic EL materials.

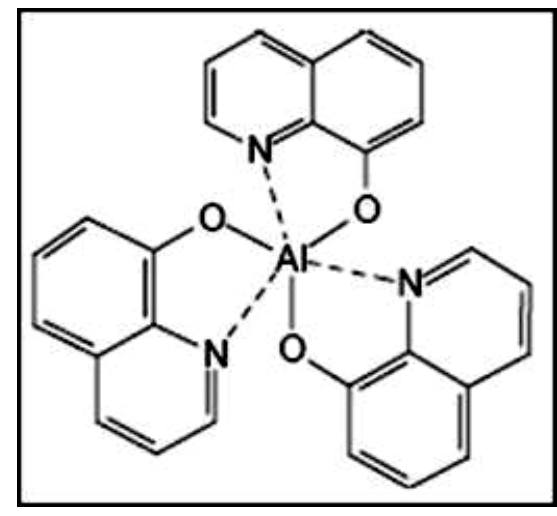

Figure 3. Molecular structure of $\mathrm{Alq}_{3}$
Among organic small molecule electroluminescent materials, 1,3,5-tris (diarylamino) benzene compounds have been researched extensively. These compounds are easily oxidized, and viewed as a potential hole transport material. Karthikeyan et al. [30] synthesized five new 1,3,5tris(diarylamino)benzene compounds. These materials are excellent in hole transport, due to their high energy level of highest occupied molecular orbital (HOMO), and high glass transition temperature. Two of them can emit blue and green lights. Recently, triplet state light emission is realized by doping phosphorescent dye into small molecules like $\mathrm{Alq}_{3}$ and 4,4'-bis (N-carbazolyl)-1,1'-biphenyl (CBP). The resulting light-emitting devices can achieve a quantum efficiency as high as $13.7 \%$ and $38.31 \mathrm{~m} / \mathrm{W}$.

\subsubsection{Polymer light-emitting materials}

The development of organic small molecule EL materials is still underway. But the crystallization, a common phenomenon among small molecules, could shorten the service life of EL devices. In addition, the films of organic small molecule EL materials are mainly formed through vacuum evaporation. To ensure light emission efficiency, the devices often adopt a multilayer structure. This brings difficulty to device assembly. It is costly to produce a large display with such materials. Therefore, many scholars have shifted their attention to polymers with excellent physical properties. These polymers could be easily prepared into large displays, thanks to the following superiorities: most polymers are very flexible, highly formable, and unlikely to crystallize; the chain conjugated polymers have a one-dimensional (1D) structure, and a band gap equivalent to the energy of visible light; soluble polymers boast good mechanical properties and film-forming property.

At present, polymer materials are used in three forms in LEDs: (1) conjugated polymers are adopted as the LEL; (2) polymers are adopted as the carrier transport layer, and small molecule EL materials as the LEL; (3) dye-doped polymers are adopted as the LEL.

(1) Conjugated polymer electroluminescent materials: poly(p-phenylenevinylene) (PPV) and its derivatives

In 1990, Burroughes et al. [31] at The Cavendish Laboratory, University of Cambridge became the first to prepare a polymer film electroluminescent device with PPV. The device outputs bluish green lights with a direct current (DC)-driven bias voltage smaller than $14 \mathrm{~V}$, and realizes a quantum efficiency of $0.05 \%$. Subsequently, in 1992, Braum and Heerger at University of California designed a lightemitting diode from PPV and its derivatives, which can dissolve in organic solvent. With a starting voltage of $3 \mathrm{~V}$, the diode can effectively emit two kinds of lights: a green light, and an orange light. Until now, PPV remains the most concerned light-emitting polymer, and is widely expected to be commercialized. The electroluminescence performance of the polymer is extraordinarily high. Because of its high molecular weight, PPV can be prepared into high-quality films. So far, various derivatives have been developed from PPV.

In EL devices, PPV, characterized by hole conductivity, can be used for the LEL, as well as the carrier transport layer in the multilayer structure. For instance, Greenham et al. [32] prepared a two-layer LED, where the poly (2,5-di (hexyloxy) cyanoterephthalylidene) (CN PPV) with high electronic affinity serves as the LEL, and PPV as the HTL. The quantum efficiency of the LED is up to $4 \%$. The growing electronic affinity of polymers can suppress the energy barrier during 
electron injection. Therefore, EL materials with different luminescent colors and light-emitting properties can be obtained through proper chemical modification of polymers. Doi et al. [33] explored the influence of the chain length of the same type of substituents over the alkoxy-substituted PPV (RO PPV), and observed that the electroluminescence intensity of the device first increased with the chain length, reached the peak when the $\mathrm{R}$ group is $\mathrm{n}$-alkyl with ten carbon atoms, and decreased with further growth in chain length. Recently, some researchers introduced Si alkyl group into the side chain of PPV, and changed the distribution of $\sigma$ key and $\pi$ bond. In this way, they effectively limited the electron distribution on the polymer chain, and realized high quantum efficiency. The obtained silane-substituted PPV has good quantum efficiency, solubility, and film-forming property.

(2) Other electroluminescent conjugated polymer materials

Other than PPV, polythiophene is another popular class of electroluminescent heterocyclic polymer materials. The electronic energy level of polythiophene, with thiophene as the skeleton, can be easily adjusted through side chain modification. The diverse spatial configurations that ensue bring unexpected electronic properties, which catches the eyes of many researchers. Polyfluorene is another type of widely studied conjugated polymers. This material can dissolve well in ordinary organic solvents, and fuse at a low temperature. With a band gap larger than $290 \mathrm{eV}$, polyfluorene provides a desirable material for blue diodes. It is one of the few blue light-emitting materials that could be applied in practice. However, the current crowding effect might be the result of the formation of low-level aggregates between the chain. Therefore, the luminescence spectrum of polyfluorene has a long tail, that is, the color is neither pure nor stable. The above copolymers can emit lights from blue to green wavelength, and have good solubility and thermal stability. The copolymer of fluorene and anthracene is very thermostat, and that of fluorene and aniline can enhance the hole transport ability, reducing working voltage. In addition, the copolymer of fluorene and carbazole (hole transport unit) can reduce the driving voltage, and cut down the working voltage by half, without any significant loss of light-emitting efficiency.

The Dow Chemical Company have studied a wide range of the homopolymers and copolymers of fluorene. They once developed a green light-emitting fluorene copolymer, and prepared it into a two-layer LED. Under the working voltage of $425 \mathrm{~V}$, the LED could reach a luminous brightness of $4,000 \mathrm{~cd} / \mathrm{m}^{2}$, and an efficiency of $7791 \mathrm{~m} / \mathrm{W}$. The company also designed a red light-emitting copolymer, whose efficiency is as high as $1 \mathrm{~lm} / \mathrm{W}$. Poly(1,4-phenylene) (PPP) is another blue light-emitting conjugated polymer. The stable polymer has a band gap of about $3 \mathrm{eV}$, which meets the requirements for blue light emission. It is an important material for blue lightemitting devices. Some researchers prepared LEDs with PPP, and managed to emit lights of the wavelength $415 \mathrm{~nm}$. Later, several PPP derivatives were adopted to prepare LEDs. These derivatives have a high fluorescence quantum efficiency. Nevertheless, the EL devices made of them face a rather low quantum efficiency. Y. Yang synthetized three soluble PPP derivatives: poly [2- (2'-ethylhexyloxy)-1,4-phenylene] (EHO-PPP), poly (2-decyloxy-1,4-phenylene) (DO-PPP), and poly [2- (6'-cyano-6'-methylheptyloxy)-1,4-phenylene] (CNPPP). Taking the ITO as the anode, and $\mathrm{Ca}$ as the cathode, the devices made of the derivatives emitted lights of the wavelength $420 \mathrm{~nm}$, and achieved the quantum efficiency of 1 $3 \%$. With air-stable cathode materials like $\mathrm{Ag}, \mathrm{In}, \mathrm{Al}$ and $\mathrm{Cu}$, the quantum efficiency of the devices fell between $0.3 \%$ and $0.8 \%$ [34].

\subsection{Dye-doped polymers / sensitized materials}

The color of the lights emitted by OLED devices is adjustable. One of the best adjustment strategies is to dope a small amount of photoluminescent dye in OLEDs. The OLEDs doped with photoluminescent dye can emit lights across the spectrum of visible light, and work efficiently for a long time. In the devices, the photoluminescent dye intercepts the spectrum of the excitons produced in OLEDs, and emits lights in its own spectrum. The doping of the dye can significantly enhance the light-emitting efficiency of the OLEDs. Besides, the dye-doped devices will have a narrow electroluminescence spectrum, i.e., emit purer lights. This is very advantageous for full-color display.

The triplet state light emission of organic small molecules is now a hot topic. Doping triplet state light-emitting small molecules into a polymer can transfer the energy from the polymer to the molecules, and realize triplet state light emission. Thus, the light-emitting efficiency of the polymer is greatly improved. Judging by the luminescence spectrum of devices, the energy of PVK can be transferred to $\operatorname{Ir}(\mathrm{PPY})_{3}$, enabling triplet state light emission. Such devices combine the efficient triplet state light emission with the good processability of polymers, and are worthy of further research.

\section{PREPARATION TECHNOLOGY OF OLED DEVICES}

According to the preparation purposes, the preparation technologies of OLED devices can be divided into the singleand multi-layer film forming technology of panel lightemitting devices, the forming technology of lattice lightemitting devices, and the forming technology of full-color devices composed of multiple light-emitting points. In addition, it is necessary to consider some factors of the preparation technologies that affect the light-emitting efficiency and service life of devices, in order to satisfy the needs of actual applications.

\subsection{Forming methods of panel electroluminescent materials}

(1) Vacuum evaporation film-forming

Vacuum coating is suitable for the film formation of substances that are easy to sublimate under high vacuum. The film is formed in a vacuum system. The coating material is placed under a high temperature to be sublimated in vacuum. The sublimated material will deposit uniformly on the lowtemperature substrate, forming a thin film. This method requires special equipment, and good thermal stability of filmforming material, which does not decompose under the sublimation temperature. The film thickness depends on the sublimation rate and evaporation time, while the sublimate rate depends on the temperature and vacuum degree. That is why temperature and vacuum degree are important industrial parameters.

(2) Dip coating or spin coating film-forming

Dip coating or spin coating is a solution film-forming method. Before coating, the film-forming material needs to be dissolved in a solvent to create a solution with a suitable 
concentration. During dip coating, the ITO electrode is immersed in the film-forming solution, and then taken out to let the solvent volatilize; Next, the solute is deposited into a film. The thickness of the film can be controlled by adjusting the concentration and viscosity of the solution, or by regulating the number of dips. Dip coating is simple and easy to implement, without needing complex instruments or equipment. However, this approach does not apply to multilayer electroluminescent devices, because the dipcoating of the second layer often leads to short-circuit and other faults to the first layer. Besides, it is difficult to control the thickness and the uniformity of the film. Spin coating is an improved version of dip coating. During spin coating, a film of uniform thickness is formed under the centrifugal force generated from substrate rotation. Specifically, the solution of the film-forming material is dripped to the center of the surface of the ITO glass electrode. Under the centrifugal force, the excess solution is thrown out. The remaining solution is adsorbed on the electrode surface, forming a uniform film. Due to the short contact time, the electrode and solution have a limited mutual influence. Therefore, spin coating can be applied to prepare multilayer devices. The defect of this technology is the difficulty in producing large films. Dip coating and spin coting require the film-forming material to be soluble in some solvents. Otherwise, it is impossible to obtain a solution of the suitable concentration. These methods are not widely applicable, owing to the poor solubility of some electroluminescent polymer materials.

(3) In-situ polymerization film-forming

In-situ polymerization directly polymerize monomers on the substrate to form a film. This is the only feasible and ideal approach to create films on electroluminescent materials with poor solubility or thermal stability. In-situ polymerization combines the pre-synthetized monomeric compounds with other polymerization reagents into a reaction system. The general flow is as follows: First, prepare a polymerization monomer reaction solution; Next, induce the polymerization reaction by electrochemical, photochemical and other methods, which directly forms a polymer film on the substrate. Through in-situ polymerization, the LEL and charge transport layers can be formed in-situ on the ITO electrode. Since the ITO electrode acts as a conductive substrate, it is quite common to prepare the LEL and charge transport layers through chemical in-situ polymerization. To ensure the progress of the electrochemical reaction, the electrolyte should be included in the monomer solution to drive the polymerization reaction at the three-electrode or two-electrode potential. As the active group of electrochemical polymerization, the double bonds of the terminal group can be introduced to the active structure to form a polymer film with a main chain of saturated alkanes. Moreover, a polymer film with a main-chain conjugated structure can be generated through oxidative electrochemical polymerization, using aromatic structure or the derivatives of pyrrole or thiophene structure as monomers. One of the prominent features of this technology is the applicability to electroluminescent polymer materials with poor solubility and biochemical properties, especially conjugated polymers. During electrochemical polymerization, the film thickness can be controlled by the electrolysis time and electrolysis voltage. In addition, the thin films prepared by this approach have very few defects. They are promising materials for ultrathin LELs. For electroluminescent devices, a thin LEL means a low starting voltage. However, postprocessing is necessary to eliminate the ionic impurities brought by electrochemical polymerization.

(4) Ion sputtering form-formation

Ion sputtering uses high-energy ions with a certain kinetic energy to bombard the film-forming material. Then, the material sputters onto the substrate, forming a film. This technology is mainly adopted to prepare charge injection layers. For the electron injection electrode, the films are mainly formed through vacuum evaporation of alkaline earths with a low work function or their alloys. For those electrode materials that are not easy to sublime, the bombardment with high-energy ion current can create a film with a preset thickness. Under the bombardment of high-energy ions, organics are prone to destructive structural changes, and thus unsuitable for preparing common charge transport layers or LEL. This technology is mainly adopted for lab research. It needs to be improved to realize industrial application.

\subsection{Latticed or graphic forming of electroluminescent materials}

An important application field of organic electroluminescent materials is the preparation of large flat displays. In all modern display devices, the display elements are arranged as a lattice. To improve the display quality, there is a continuous increase of resolution. This calls for a huge number of refined display elements. Apparently, the previously mentioned film-forming technologies cannot meet the production efficiency or product quality. The latticed forming problem must be solved to develop image display devices with organic electroluminescent materials. At present, this problem is mainly solved by the following processing techniques:

(1) Computer-controlled inkjet printing

This method is developed from dip coating. The first step is to dissolve organic electroluminescent materials in a solvent to prepare a printing solution (ink). Then, the solution is placed in a machine with inkjet printing function. The computercontrolled machine will print a functional film with a specific graphic or latticed structure on the substrate. The size and time of droplets ejected by the inkjet head can be controlled precisely to form precise graphic or latticed patterns on the base material, just like graphic printing. The thickness of the film can be regulated by the concentration and viscosity of the solution. Of course, as dip coating and spin coating, inkjet printing requires that the material should be highly soluble in a certain solvent, and could be prepared into LEL and charge transport layers of specific thickness and shape and uniform pixel size, under concentration control or printing technology control. This technology is known for efficient working and precise control.

\section{(2) Photolithography}

Photolithography is a mature processing technique widely used in integrated circuits, printed circuits and printed plates. In the preparation of electroluminescent devices, this technique is primarily adopted to make the anode, that is, the latticed and graphic processing of the ITO electrode. Generally, the pixels on the ITO electrode prepared by photolithography will be made through coating technologies. The cathode and the LEL are normally not prepared by photolithography. After all, the organic layers are easily damaged and polluted during the cleaning of the photoresist.

(3) Shielding cover with vacuum evaporation 
Extended from vacuum evaporation film-forming, this approach creates specific graphic or latticed LEL and charge transport layers in nonprotected areas, under the protection of a shielding cover with a specific precision structure. The shielding cover could be prepared from metals or organic materials. In principle, this method is not limited by the type of material. But the quality and performance of the prepared electroluminescent devices hinge on the mechanical strength and processing accuracy of the shield.

\section{APPLICATION AND PROBLEMS OF OLED}

OLED is mainly used in the fields of new displays, lighting, and semiconductor lasers. In recent years, the United States, the United Kingdom, Japan, Germany, the Netherlands, and Sweden have been very active in new OLED displays. Many companies invest heavily in this field, including Philips, Siemens, and Hoechst in Europe; Pioneer, Toyota, TDK, Seiko-Epson, Sumitomo, NEC, and Sanyo in Japan; Kodak, HP, IBM, and DuPont in the United States. Among them, Pioneer has piloted the commercial production of multi-color displays for dashboards and small-molecule light-emitting displays for mobile phones. LG and other companies in South Korea also begin to enter this field. Philips (the Netherlands) and Siemens (Germany) constructed one production line of polymer light-emitting digital graphics displays for mobile phones in Hellen and Malaysia, respectively. Both production lines will soon be put into production. DuPont/Uniax (United States), in association with RITEK (Taiwan, China), is planning to develop a production line of polymer lightemitting displays. Many companies have launched 1/4VGA full-color display prototypes based on small molecule lightemitting diodes.

Semiconductor lasers are widely used in modern science and technology. Compared with traditional inorganic semiconductors, organic semiconductors have the advantages of easy processing, low cost, and flexibility. Therefore, electrically-driven lasers prepared with organic semiconductors have a wide application prospect. Lightexcited laser and amplified spontaneous emission are ubiquitous in semiconductor polymers, organic small molecules, and organic single crystals. Semiconductor polymers, organic small molecules, and organic single crystals have been successfully applied in thin-film organic lightemitting devices. Schon et al. [35] of Bell Laboratories prepared organic electric pumped laser devices with tetracene single crystals. When the injection current reaches a critical density, the emission spectrum will become narrower, from the original $120 \% \mathrm{eV}$ to less than $1 \% \mathrm{eV}$. When the gain exceeds the loss, a net gain laser will be produced. The reason for their success lies in the use of high-mobility organic single crystals. Unlike amorphous organic materials, these single crystals have a very limited effect of charge-induced absorption. In addition, the field effect transistor (FET) was adopted to inject electrons and holes, reducing the loss of metal electrodes. However, polymer electric pumped laser devices have not been materialized yet.

Further research of organic and polymer electroluminescent devices will focus on the following problems:

(1) Basic research on light-emitting mechanism (improving internal quantum efficiency): phosphorescence and other triplet state light-emitting materials;

(2) Materials and devices of large full-color panel displays;
(3) Improving the service life of light-emitting devices: identifying the key factors affecting the service life, and developing corresponding solutions;

(4) Organic/polymer lasers.

\section{ACKNOWLEDGMENT}

This work was supported by the National Natural Science Foundation of China Joint Fund for Cross-strait Scientific and Technological Cooperation (U2005212), Natural Science Foundation of China (51702272), Support and training program for top young talents of "young eagle program" in Fujian Province (2020), Natural Science Foundation of Fujian Province of China (2019J01873, 2020J01290 and 2020J02049), Major Project of Science and technology of Xiamen City (3502ZCQ20201001 and 3502Z20201003) and Science and technology projects of Xiamen Institute of Technology (YKJ20001R, XPDKT20008 and XPDKQ20002), Open fund of Fujian Provincial Key Laboratory of Functional Materials and Applications, Xiamen University of Technology (Grant No. fma2020009), Graduate science and technology innovation project(YKJCX2020007).

\section{REFERENCES}

[1] Lin, K.B., Xing, J., Quan, L.N., De.Arquer, F.P.G., Gong, X.W., Lu, J.X., Xie, L.Q., Zhao, W.J., Zhang, D., Yan, C.Z., Li, W.Q., Liu, X.Y., Lu, Y., Kirman, J., Sargent, E.H., Xiong, Q.H., Wei, Z.H. (2018). Perovskite lightemitting diodes with external quantum efficiency exceeding 20 per cent. Nature, 562(7726): 245-248. https://doi.org/10.1038/s41586-018-0575-3

[2] Xin, H.S., Ge, C.W., Jiao, X.C., Yang, X.D., Rundel, K., McNeill, C.R., Gao, X.K. (2018). Incorporation of 2,6connected azulene units into the backbone of conjugated polymers: Towards high-performance organic optoelectronic materials. Angewandte Chemie International Edition, 57(5): 1322-1326. https://doi.org/10.1002/anie.201711802

[3] Tada, A., Geng, Y.F., Wei, Q.S., Hashimoto, K., Tajima, K. (2011). Tailoring organic heterojunction interfaces in bilayer polymer photovoltaic devices. Nature Materials, 10(6): 450-455. https://doi.org/10.1038/NMAT3026

[4] Yao, Y.J., Zhao, Q., Wei, W., Chen, Z., Zhu, Y., Zhang, P., Zhang, Z.T., Gao, Y.F. (2020). WO3 quantum-dots electrochromism. Nano Energy, 68: 104350. https://doi.org/10.1016/j.nanoen.2019.104350

[5] Yokota, T., Kajitani, T., Shidachi, R., Tokuhara, T., Kaltenbrunner, M., Shoji, Y., Ishiwari, F., Sekitani, T., Fukushima, T., Someya, T. (2018). A few-layer molecular film on polymer substrates to enhance the performance of organic devices. Nature Nanotechnology, 13(2): 139-144. https://doi.org/10.1038/s41565-0170018-6

[6] Behzad, S.K., Najafi, E., Amini, M.M., Janghouri, M., Mohajerani, E., Ng, S.W. (2014). Yellow-green electroluminescence of samarium complexes of 8hydroxyquinoline. Journal of Luminescence, 156: 219228. https://doi.org/10.1016/j.jlumin.2014.08.013

[7] Ghosh, S., Shankar, S., Philips, D.S., Ajayaghosh, A. (2020). Diketopyrrolopyrrole-based functional supramolecular polymers: Next-generation materials for 
optoelectronic applications. Materials Today Chemistry, 16: 100242 .

https://doi.org/10.1016/j.mtchem.2020.100242

[8] An, K.B., Xie, G.H., Gong, S.L., Chen, Z.X., Zhou, X., Ni, F., Yang, C.L. (2020). Monoradically luminescent polymers by a super acid-catalyzed polymerization and deep-red electroluminescence. Science China (Chemistry), 63(9): 1214-1220. https://doi.org/10.1007/s11426-020-9778-9

[9] Marrani, A.G., Novelli, V., Sheehan, S., Dowling, D.P., Dini, D. (2014). Probing the redox states at the surface of electroactive nanoporous $\mathrm{NiO}$ thin films. ACS Applied Materials \& Interfaces, 6(1): 143-152. https://doi.org/10.1021/am403671h

[10] Zhao, W., Yang, Z.Y., Jiao, B., Wu, Z.X. (2015). Organic alternating current electroluminescence device based on 4,4'-bis(N-phenyl-1-naphthylamino) biphenyl/1,4,5,8,9,11-hexaazatriphenylene charge generation unit. Organic Electronics, 17(2): 44-55. https://doi.org/10.1016/j.orgel.2014.11.018

[11] Chen, C.J., Zhuang, Y.X., Li, X.Y., Lin, F.Y., Peng, D.F., Tu, D., Xie, A., Xie, R.J. (2021). Achieving remote stress and temperature dual-modal imaging by doublelanthanide-activated mechanoluminescent materials Advanced Functional Materials, 2101567 https://doi.org/10.1002/adfm.202101567

[12] Lee, C.C., Su, W.C., Chang, W.C., Huang, B.Y., Liu, S.W. (2015). The effect of charge transfer state on the open-circuit voltage of small-molecular organic photovoltaic devices: A comparison between the planar and bulk heterojunctions using electroluminescence characterization. Organic Electronics, 16: 1-8. https://doi.org/10.1016/j.orgel.2014.10.040

[13] Xu, W., Sun, H.C., Xu, J., Li, W., Mu, W.W., Liu, Y., Yan, M.Y., Huang, X.F., Chen, K.J. (2011). Electroluminescence with micro-watt output from ultrasmall sized $\mathrm{Si}$ quantum dots/amorphous $\mathrm{SiO}_{2}$ multilayers prepared by laser crystallization method. Applied Surface $\quad$ Science, 258(1): 346-349. https://doi.org/10.1016/j.apsusc.2011.08.062

[14] Li, Y.F., Yao, B., Deng, R., Li, B.H., Zhang, Z.Z., Shan, C.X., Zhao, D.X., Shen, D.Z. (2013). A comparative study on electroluminescence from $\mathrm{ZnO}$-based double heterojunction light emitting diodes grown on different lattice mismatch substrates. Journal of Alloys and Compounds, 575(10): 233-238. https://doi.org/10.1016/j.jallcom.2013.04.036

[15] Chen, D.Y., Wang, Y.Y., Sun, Y., He, Y.J., Zhang, G. (2015). Effect of resonant tunneling on electroluminescence in nc-Si $/ \mathrm{SiO}_{2}$ multilayers-based $\mathrm{p}$-in structure. Thin Solid Films, 576(2): 19-22. https://doi.org/10.1016/j.tsf.2014.12.035

[16] Hu, Z.H., Wang, Q., Zhong, Z.M., Chen, Y.W., Peng, J.B., Wang, J., Cao, Y. (2018). Solution processed alkalimetal and alkaline-earth-metal compounds as the efficient electron injection layer in organic light-emitting diodes. Synthetic Metals, 236: 31-35. https://doi.org/10.1016/j.synthmet.2017.12.010

[17] Wang, Y.Y., Yu, Z.M., Yang, Y., Sun, J.M. (2021). Bright red electroluminescence from $\mathrm{Al}_{2} \mathrm{O}_{3} / \mathrm{Eu}_{2} \mathrm{O}_{3}$ nanolaminate films fabricated by atomic layer deposition on silicon. Scripta Materialia, 196: 113750. https://doi.org/10.1016/j.scriptamat.2021.113750

[18] Cheon, H.J., Joo, C.W., Lee, D.Y., Huseynova, G., Lee,
J.H., Lee, J., Kim, Y.H. (2021). Highly efficient orange phosphorescent organic light-emitting diodes with (4(3,5-dimethylphenyl)-2-(m-tolyl)pyridine)-based iridium complex. Dyes and Pigments, 186: 109006. https://doi.org/10.1016/j.dyepig.2020.109006

[19] Dayan, S. (2021). Performance improvement of $\mathrm{Co}_{3} \mathrm{O}_{4} @$ nHAP hybrid nanomaterial in the UV lightsupported degradation of organic pollutants and photovoltaics as counter electrode. Journal of Molecular Structure, $1238: 130390$. https://doi.org/10.1016/j.molstruc.2021.130390

[20] Deng, L.L., Zhou, Z.J., Jia, B.L., Zhou, H.W., Peng, L., Shang, W.J., Feng, J., Chen, S.F. (2018). Improved electron injection and efficiency in blue organic lightemitting diodes using coupled electric field near cathode. Organic Electronics, 53: 346-352. https://doi.org/10.1016/j.orgel.2017.12.004

[21] Li, R.Q., Wang, J., Qin, Y., Liu, C.Y., Wang, Y.Z., Zhang, X.W., Xu, Y.N., Chen, Y.H., Lai, W.Y., Li, Y.H., Huang, W. (2020). Inverted organic light-emitting devices using a charge-generation unit as an electron injector. Organic Electronics, 76: 105445. https://doi.org/10.1016/j.orgel.2019.105445

[22] Zhao, H., Ding, H.Y., Li, S.J., Liu, M., Yang, J.L., Zhao, Y.L., Pan, N., Wang, X.P. (2019). Improving electron injection in all-inorganic perovskite light-emitting diode via electron transport layer modulation. Optik, 191: 6874. https://doi.org/10.1016/j.ijleo.2019.05.106

[23] Mammana, S.S., Greatti, A., Luiz, F.H., Da Costa, F.I., Mammana, A.P., Calligaris, G.A., Cardoso, L.P., Mammana, C.I.Z., Den Engelsen, D. (2014). Study of wet etching thin films of indium tin oxide in oxalic acid by monitoring the resistance. Thin Solid Films, 567: 2031. https://doi.org/10.1016/j.tsf.2014.07.027

[24] Zhang, Y.J., Sims, M., Li, S., Aziz, H. (2017). Tripletinduced degradation: An important consideration in the design of solution-processed hole injection materials for organic light-emitting devices. Organic Electronics, 48: 217-222. https://doi.org/10.1016/j.orgel.2017.05.045

[25] Kim, G.W., Lampande, R., Choe, D.C., Bae, H.W., Kwon, J.H. (2015). Efficient hole injection material for low operating voltage blue fluorescent organic light emitting diodes. Thin Solid Films, 589: 105-110. https://doi.org/10.1016/j.tsf.2015.04.076

[26] Zhang, L.P., Li, D.M., Li, X.B., Wang, B., Xu, G.P., Zhao, X., Xia, G.L., Gavrilyuk, A., Zhao, J.P., Li, Y. (2019). Further explore on the behaviors of IR electrochromism of a double layer constructed by proton acid-doped polyaniline film and ITO layer. Dyes and Pigments, 170 : 107570. https://doi.org/10.1016/j.dyepig.2019.107570

[27] Yang, P.P., Chen, J.F., Huang, Z.H., Zhan, S.M., Jiang, Z.J., Qiu, Y.Q., Shao, C. (2009). A novel 1D organic optic-electronic nanomaterial using PPV (p-type) as shell and Alq3 (n-type) as core. Materials Letters, 63(23): 1978-1980. https://doi.org/10.1016/j.matlet.2009.06.021

[28] Yang, K.S., Shin, H.K., Kim, C., Kwon, Y.S. (2005). Photoluminescent and electroluminescent investigations of OLEDs using Alq2-Nq. Colloids and Surfaces A: Physicochemical and Engineering Aspects, 257-258: 6366. https://doi.org/10.1016/j.colsurfa.2004.07.039

[29] Chai, W.M., Zhu, W.D., Chen, D.D., Chen, D.Z., Xi, H., Chang, J.J., Zhang, J.C., Zhang, C.F, Hao, Y. (2020). Combustion-processed $\mathrm{NiO} / \mathrm{ALD} \mathrm{TiO}_{2}$ bilayer as a novel 
low-temperature electron transporting material for efficient all-inorganic $\mathrm{CsPbIBr} 2$ solar cell. Solar Energy, 203:

$10-18$.

https://doi.org/10.1016/j.solener.2020.04.024

[30] Karthikeyan, C. S., Wietasch, H., Thelakkat, M. (2007). Highly efficient solid-state dye-sensitized $\mathrm{TiO}_{2}$ solar cells using donor-antenna dyes capable of multistep charge-transfer cascades. Advanced Materials, 19(8): 1091-1095. https://doi.org/10.1002/adma.200601872

[31] Burroughes, J.H., Bradley, D., Brown, A.R.N., Marks, R.N., Mackay, K.D. (1990). Light-emitting diodes based on conjugated polymers. Nature, 347(6293): 539-541. https://doi.org/10.1038/347539a0

[32] Greenham, N.C., Moratti, S.C., Bradley, D.D.C., Friend, R.H. Holmes, A.B. (1993). Efficient light-emitting diodes based on polymers with high electron affinities.
Nature, 365: 628-630. https://doi.org/10.1038/365628a0

[33] Doi, S., Shirai, N., Sato, Y. (1997). ChemInform Abstract: Abnormal products in the bischler-napieralski isoquinoline synthesis. ChemInform, 28(50). https://doi.org/10.1002/chin.199750152

[34] Yang, Y., Holdcroft, S. (2005). Synthetic strategies for controlling the morphology of proton conducting polymer membranes. Fuel Cells, 5(2): 171-186. https://doi.org/10.1002/fuce.200400058

[35] Schn, J.H., Baumgartner, F.P., Arushanov, E., Riazinejad, H. (1996). Photoluminescence and electrical properties of $\mathrm{Sn}$ - doped $\mathrm{CuGaSe} 2$ single crystals. Journal of Applied Physics, 79(9): 6961-6965. https://doi.org/10.1063/1.361460 